\title{
Design for social interaction through physical play in diverse contexts of use
}

\author{
Tilde Bekker $\cdot$ Janienke Sturm $\cdot$ Emilia Barakova
}

Published online: 17 December 2009

(c) The Author(s) 2009. This article is published with open access at Springerlink.com

\begin{abstract}
Products can support user groups in social interaction and/or physical play in various ways. Depending on the requirements and needs of specific user groups and contexts of use, different approaches are applied to create successful designs. This special issue contains papers that describe designs for children, adults and elderly for sports, home and outdoor contexts. The papers in this issue explore how technology can contribute to enhancing user experiences in terms of social interaction and physical activities. Knowledge from very diverse research areas, such as, social psychology, persuasive technology, child development, human-robot interaction, and creativity is used as an inspiration source for the various projects.
\end{abstract}

Keywords Games - Social interaction - Physical play · Tangible interaction

\section{Introduction}

This special issue is a follow-up of a workshop on social and physical play in which researchers exchanged knowledge about using theories as a basis for design in this area. Theories about human behaviour can be used as an inspiration source when creating novel concepts. In the context

T. Bekker $(\bowtie) \cdot$ J. Sturm · E. Barakova

Department of Industrial Design,

Eindhoven University of Technology, P.O. Box 513,

5600 MB Eindhoven, The Netherlands

e-mail: m.m.bekker@tue.nl

J. Sturm

e-mail: j.sturm@tue.nl

E. Barakova

e-mail: e.i.barakova@tue.nl of designing for social and physical play a wide range of theories have been examined to inform design directions and decisions. In this special issue design concepts are described from diverse research areas and are intended for various user groups.

The work covered in the special issue is inspired by theories from diverse areas. A number of references are made to theories about social interaction and collaboration. For example, the theory by Broadhead [2] about early play and learning describes how children's communication also develops from non-reciprocal to more reciprocal actions and language when being able to play in a more collaborative manner. Other relevant concepts that were mentioned as being indicators of social communication were for example turn-taking, imitation, joint attention, shared gaze, expressing and recognizing emotions.

The papers also provide theories related to physical interaction. The work by Lakoff and Johnson [6] is used to understand how our bodies are the basis for how we build up our experiences and interactions with our environment. Ideas about kinesthetic empathy by Fogtmann et al. [4] and kinesiology (the science or study of (human) movement) are used as input to look into how people can learn and explore through their movements. The work by Bruner [3] and his ideas about enactive knowledge (which is the knowledge stored in the form of motor responses and acquired by the act of "doing") is also mentioned in the context of thinking about physical interaction. The theory can help to explore different types of physical interaction styles.

\section{Research areas}

Diverse research areas look into aspects of social interaction and physical play. Social and physical interaction is 
also a theme that plays in role in the relatively new area of tangible interaction. Four themes, tangible manipulation, spatial interaction, embodied facilitation and expressive representation, described in the framework by Hornecker and Buur [5], nicely illustrate this. Two of them, namely embodied facilitation and spatial interaction, are most closely related to our workshop topic because they are related to social and often also to physical interaction. The projects by Bekker et al. and by Leal Penados et al. explore indoor and outdoor mobile solutions using sensor-actuator technology to create interactive tangible play objects. Lentini and Decortis apply low-tech tangible camera tools for exploring the environment.

The area of exertion interfaces studies interfaces that require a form of physical exercise. For example, the paper by Mueller et al. describes networked sport-like games to stimulate social bonding. They use ideas about designing for social interaction to make physical activities more appealing. The paper by Abeele and De Schutter focuses on indoor computer games, that are controlled by the Wii-mote (using motion sensors; http://www.wii.com)

Pervasive games combine social interaction and physical play. These games are often based on existing computer games, but are enhanced by adding a physical and networked dimension. For example, the mixed-reality game Uncle Roy All Around You [1] integrates aspects of the physical world (a city) and a virtual game world: street players and online players must work together using web cams, audio and text messages to find a secret destination. The paper by Soute and Markopoulos describes a number of outdoor pervasive games, combining the opportunities of outdoor traditional games with characteristics of indoor digital games, requiring physical activity and social interaction.

Social play is becoming an increasingly important topic in the field of robotics. Robots can be used to train social and emotional skills in a playful manner. For example, Paro [7] is a robot seal designed to perform emotive and attentive exchange with humans in the simplest and most comprehensive way. Paro is used to study whether robot pets can be used for therapeutic purposes, such as contributing to social interaction. In the paper by Wainer et al. autistic children receive classes about programming Lego robots in which they are stimulated to collaborate while creating their Lego programs. In the paper by Barakova and Lourens autistic children are motivated to collaborate by jointly having to interact with a life-size controller that interacts with a robot in a storytelling context.

\section{User groups}

Interactive products that stimulate social and physical play can be relevant for various user groups for different reasons, for example for children and adults to prevent obesity, for elderly to avoid loneliness or for people with special needs to train specific social and physical skills. Abeele and De Schutter describe a design project of an intergenerational game controlled with the Wiimote which is designed both for children and elderly users. Both Wainer et al. and Barakova and Lourens describe studies in which robots are used to stimulate interaction between autistic children.

The work by Bekker et al. describes various design cases of intelligent play objects for children that stimulate social interaction through the negotiation of game rules and goals created by the children themselves. The paper by Soute and Markopoulos describes the design of various pervasive games for children. The project by Leal Panados et al. describes the design process of an intelligent cuddly toy. Children have to care for their toy through diverse physical and social interactions and are rewarded for good behaviour with a positive effect on the cuddly toy's health.

Other papers describe projects for adult users. For example, the work by Lentini and Decortis describes how the use of low-tech camera-like tools are used to stimulate people to explore their environment both through creating photos and a discussion about the artifacts created with the pinhole cameras. The work by Mueller et al. on 'Table Tennis for Three' explores how to apply technology to design a distributed exertion game for adult users.

\section{Conclusion}

Social and physical interactions have been explored in relation to diverse aspects of everyday life. The papers presented in this special issue explore the opportunities that new technologies offer not just for individual entertainment, but rather to enhance the benefits of physical and social interactions. They describe a wide diversity of work in terms of the technologies applied, such as robots, computer games, cuddly toys and low-tech camera tools to enhance various activities such as exploring the environment, participating in sports activities, education, playing physical computer games and being connected to other people.

Open Access This article is distributed under the terms of the Creative Commons Attribution Noncommercial License which permits any noncommercial use, distribution, and reproduction in any medium, provided the original author(s) and source are credited.

\section{References}

1. Benford S (2005) Pushing the boundaries of interaction in public. Interactions 12(4):57-58

2. Broadhead P (2004) Early years play and learning: developing social skills and cooperation. RoutledgeFalmer, London 
3. Bruner J (1968) Processes of cognitive growth: infancy. Clark University Press, Worcester

4. Fogtmann MH, Fritsch J, Kortbek KJ (2008) Kinesthetic interaction: revealing the bodily potential in interaction design. In: Proceedings of OZCHI '08. ACM, NY, pp 89-96

5. Hornecker E, Buur J (2006) Getting a grip on tangible interaction: a framework on physical space and social interaction. In: Proceedings of CHI '06. ACM, NY, pp 437-446
6. Lakoff G, Johnson M (1999) Philosophy in the flesh. Basic Books, New York

7. Shibata T, Yoshida M, Yamato J (1997) Artificial emotional creature for human-machine interaction. In: IEEE International Conference on systems, man and cybernetics, vol 3. IEEE, 1997, pp 2269-2274 